\title{
ANALYSIS OF LOSS WATER ON PIPE DISTRIBUTION "NON REVENUE WATER (NRW)” NETWORK PDAM SURYA SEMBADA CITY OF SURABAYA WITH CAUSAL LOOP DIAGRAM (CLD)
}

\author{
Yanatra Budi Pramana*) \\ ${ }^{*}$ Industrial Engineering Department, Faculty of Industrial Technology \\ University of PGRI AdiBuana Surabaya \\ Email : p_yanatra@unipasby.ac.id
}

\begin{abstract}
Water is an important supporting factor in humanlife. The problem of clean water in an area is managed by Perusahaan Daerah Air Minum (PDAM) Surabaya. PDAM Surya Sembada as a water management company in Surabaya is expected to be able to distributethe needs of clean water, however, in the distribution network, sometimes experiencing problems that cause water is not fully channeled to the customer, to overcome this problem, the Causal Loop Diagram approach is used to identify the cause of the water loss, after knowing these factors, we have been made model with Causal Loop model,to calculate the water loss that occurred, in PDAM Surya Sembada Surabaya especially DMA 109 using Infrastructure Leakage Index (ILI) method. The Causal Loop Diagram approach, various causes of water loss include: physical water loss caused by reservoir leakage, transmission line leakage and distribution pipeline leakage; non physical water loss caused by meter fault, wild connection and water theft. Based on the data obtained, obtained NRW value at DMA 109 of $439.455 .72 \mathrm{~m}^{3} /$ year or by $25 \%$ with a loss of $329.591 .8 \mathrm{~m}^{3}$ / year. Service pipe leakage up to the customer meter is $1.757 .822 .8 \mathrm{~m}^{3} /$ year.

Keywords:Causal Loop Diagram, Distribution Network Pipeline, Infrastructure Leakage Index (ILI), Water Loss
\end{abstract}

\section{Introduction}

Clean water is an important supporting factor for human survival. The need for raw water for various purposes, especially clean water for households, public places, industries and others will continue to increase from time to time in line with the pace of development in various sectors and fields and the number of population continues to grow, expected to be able to distribute and meet the needs of clean water community well and evenly with the existing pipeline distribution system, if the distribution system is not good enough, will cause various problems such as lack of water pressure so that the flow of water is not distributed evenly. Efforts to reduce the level of water leak can be done by first monitoring the condition of water that is distributed, both cubic water and pressure.

Monitoring efforts will be easier and more accurate if done in a small area, in other words divide the vast area into small parts. To maintain water availability, PDAM utilizes technology by building District Meter Area (DMA) and renewal of old pipes that reduce pipe leakage. Technology is the latest knowledge of knowledge and practice skills in the production of consumption and distribution of products and services in the development process(Suwarno and Nurcahyanie, 2007).

Umar et al., 2014, discusses the distribution system in predicting the magnitude and location of the leak using only one input data, namely pressure and using the method of Extreme Learning Machine. While in research writer use more than one input data and use method of Infrastructure Leakage Index (ILI). Ardiansyah et al., 2005 discussed about the water distribution performance system by entering different data with data presented by the author with different processing also.

Therefore, it is necessary to analyze the water losses in the pipelines of PDAM Surya Sembada Surabaya using the Causal Loop Diagram (CLD) approach and the Infrastructure Leakage Index (ILI) method to 
TiBuana

Journal of applied Industrial Engineering-University of PGRI AdiBuana

determine the water loss and how to overcome it.

\section{Methods}

This research was conducted at PDAM Surya Sembada Kota Surabaya. The method used in this research is Causal Loop Diagram
(CLD) and Infrastructure Leakage Index (ILI) approach. Data were analyzed by Causal Loop Diagram (CLD) approach and Infrastructure Leakage Index (ILI) method with the following steps:

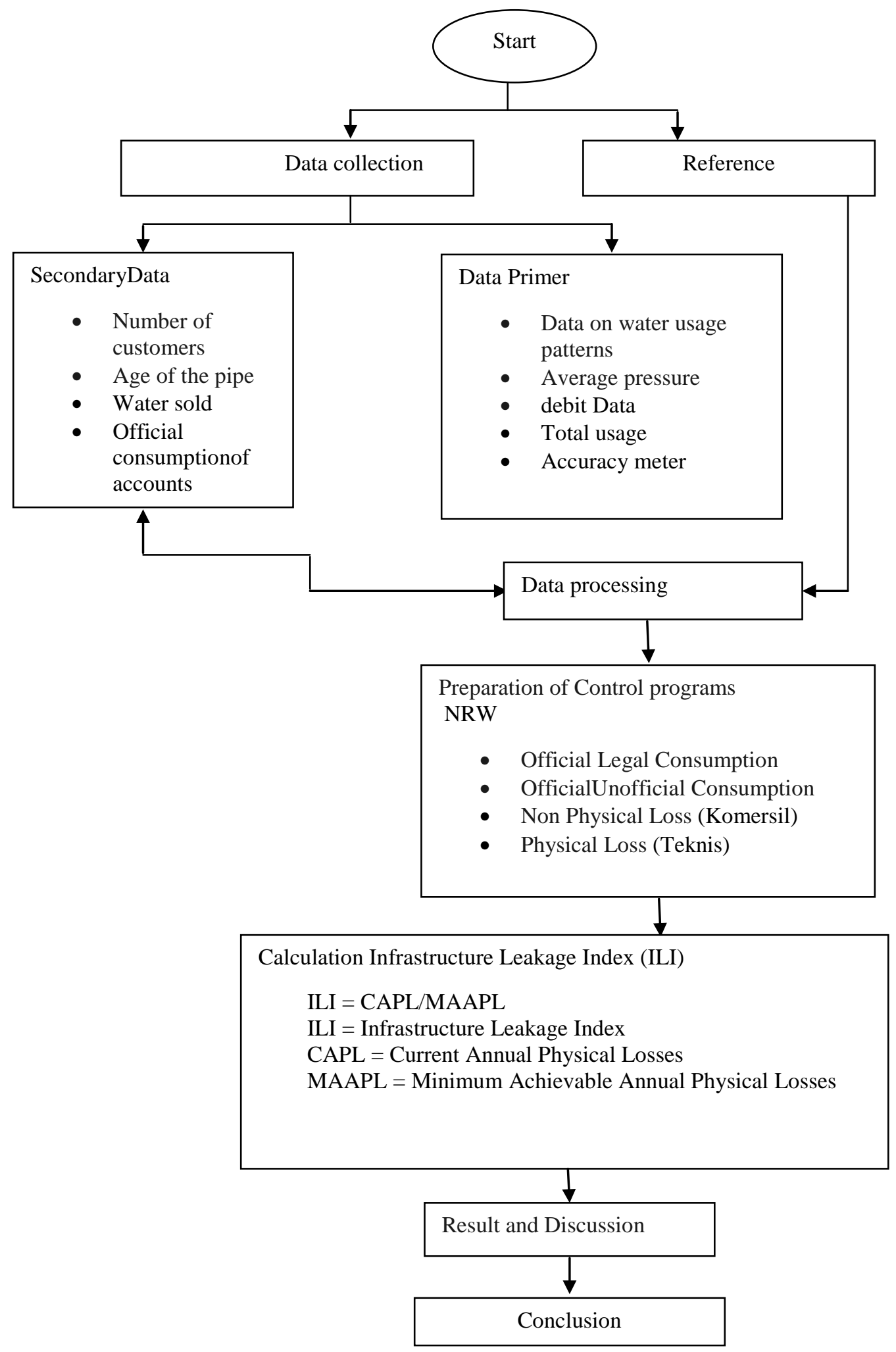

TiBuana, Vol. 01, No. 1, 2018| 25 
Figure 1. Flowchart model

\section{Causal Loop}

To search and investigate the location of sources of water loss in terms of initiative taking is as follows :

1. Passive investigation

2. Active investigation include:

Experiment to detect and measure

1. Pressure Control

2. Zone Metering

3. Distrik Metering

4. Waste Metering

5. Calculate MAAPL
6. Calculate CAPL

7. Calculate ILI (CAPL/MAAPL)

8. Adjustment For Intermittent Supply

9. Sounding regulary

10. Compare ILI with matrix study physical loss.

\section{Result and discussion}

\section{A. Causal Loop Diagram}

the modeling of causal relationship or marked "S" and "O" can be seen in the table below

Table 1.The Power of Causal Relationships

\begin{tabular}{|c|c|}
\hline Sign $S$ & Sign 0 \\
\hline $\begin{array}{l}\text { 1.Reservoir leak against: } \\
\text { - Construction damage due to cracking } \\
\text { - The installation procedure is incorrect } \\
\text { 2.Incorrect installation procesedure to: } \\
\text { - Many faulty valves and fittings } \\
\text { - Connection leakage } \\
\text { 3.Construction damage due to cracking of: } \\
\text { - Insufficient pipeline depth } \\
\text { 4. The depth of the pipe is not enough } \\
\text { - Transmission leakage } \\
\text { 5. Leakage of transmission to: } \\
\text { - Connection leakage } \\
\text { 6.Connection leakage to: } \\
\text { - Many pipe connections } \\
\text { - Many home connections } \\
\text { 7.Leakage of distribution pipes to: } \\
\text { - Many home connections } \\
\text { - Small, long pipes due to large areas } \\
\text { 8.Small pipe length due to large area against: } \\
\text { - Reservoir leak } \\
\text { - The installation procedure is incorrect } \\
\text { 9. Unlisted connection to: } \\
\text { - Number of pipe connections } \\
\text { 10.Error meter against: } \\
\text { - Small, long pipes due to large areas } \\
\text { - Error accuracy of irregular periods } \\
\text { 12.Wild connection to : } \\
\text { - Unlisted connection } \\
\text { - Water meters are damaged }\end{array}$ & $\begin{array}{l}\text { 1. Leakage of reservoir against } \\
\text { small pipe length due to large } \\
\text { area } \\
\text { 2. Many pipe connections to } \\
\text { incorrect installation } \\
\text { procedures } \\
\text { 3. meter error on accuracy of data } \\
\text { meter recording } \\
\text { 4. Meter bye pass against leakage } \\
\text { of distribution pipe } \\
\text { 5. Wild connection and water } \\
\text { theft against incorrect } \\
\text { installation procedure } \\
\text { 6. Water meters are damaged } \\
\text { consumers against construction } \\
\text { damage due to cracks }\end{array}$ \\
\hline
\end{tabular}




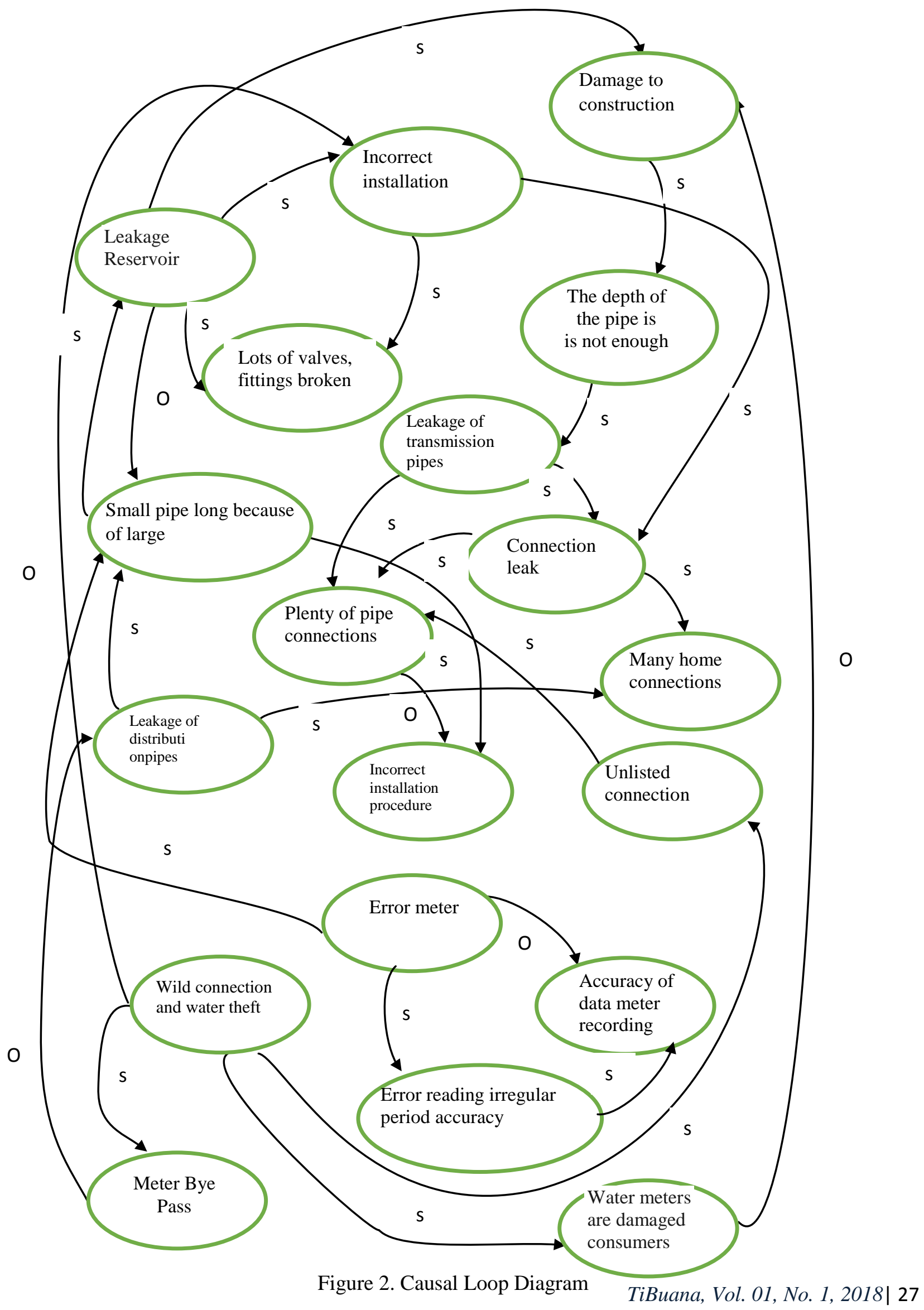


TiBuana

Journal of applied Industrial Engineering-University of PGRI Adi Buana

\section{B. Data Water discharge}

Table 2.Data water discharge

\begin{tabular}{llcc}
\hline DMA & \multicolumn{1}{c}{ Month } & $\begin{array}{c}\text { TOTAL } \\
\text { CUSTOMERS }\end{array}$ & $\begin{array}{c}\text { USE } \\
\text { ACCOUNTS } \\
\mathbf{( m}^{3} \mathbf{)}\end{array}$ \\
\hline 109 & JANUARY & 3.310 .00 & 111.127 .00 \\
& FEBRUARY & 3.313 .00 & 109.764 .00 \\
& MARCH & 3.316 .00 & 110.824 .00 \\
& APRIL & 3.320 .00 & 106.812 .00 \\
& MAY & 3.322 .00 & 108.315 .00 \\
& JUNE & 3.325 .00 & 114.518 .00 \\
& JULY & 3.323 .00 & 102.522 .00 \\
& AUGUST & 3.329 .00 & 116.437 .00 \\
& SEPTEMBER & 3.335 .00 & 100.608 .00 \\
& OKTOBER & 3.337 .00 & 107.524 .00 \\
& NOVEMBER & 3.338 .00 & 116.583 .00 \\
& DECEMBER & 3.339 .00 & 113.333 .00 \\
& & & $1.318,367.00$ \\
& & 39.907 .00 & 109.863 .3 \\
& & &
\end{tabular}

(Source : PDAM Surya Sembada Surabaya city)

\section{Pressure Data}

Tabel 3.Pressure on PDAM Surya Sembada Surabaya city

\begin{tabular}{cccc}
\hline Month & P0 (atm) & P1 (atm) & P2 (atm) \\
\hline December 2015 & 2.2 & 2.2 & 1.25 \\
January & 3.6 & 2.4 & 0.40 \\
February & 3.4 & 2.5 & 0.25 \\
March & 3.7 & 3.0 & 0.15 \\
April & 3.7 & 3.3 & 0.25 \\
May & 3.7 & 3.1 & 0.52 \\
June & 4.2 & 3.2 & 1.80 \\
July & 4.0 & 3.0 & 0.80 \\
August & 2.9 & 2.3 & 0.80 \\
October & 2.3 & 2.0 & 0.52 \\
November & 2.6 & 1.2 & 0.30 \\
December & 2.3 & 2.0 & 0.20 \\
Total & 40.5 & 31.9 & 7.64 \\
Average & & 2.04 & \\
\hline
\end{tabular}


(Source : PDAM Surya Sembada Surabaya city)

$$
\begin{aligned}
& \text { P0 }=\text { pressure at the beginning } \\
& \text { P1 }=\text { pressure at the middle } \\
& \text { P2 }=\text { pressure at the end }
\end{aligned}
$$

\section{Pattern Data water usage}

\begin{tabular}{ll}
\hline \multicolumn{2}{c}{ Table4.Pattren usage water } \\
\hline Month & Stand Meter \\
\hline December 2015 & 4261.1 \\
January & 4286.7 \\
February & 4298.4 \\
March & 4321.6 \\
April & 4350.2 \\
May & 4382.4 \\
June & 4408.9 \\
July & 4421.3 \\
August & 4453.8 \\
September & 4480 \\
October & 4500,6 \\
November & 4532.1 \\
December & 4559.6 \\
\hline
\end{tabular}

\section{E. Data Accuracy in Meter}

From the average customer as much as 3.325.58 or 3.326 home connections obtained the amount of the difference (liter) measuring glass that is 663.2 liters. This meter accuracy data will be used to calculate the percent value of water loss from customer meter.

\begin{tabular}{|c|c|c|}
\hline $\begin{array}{l}\text { Total distribution of } \\
\text { water }\left(\mathbf{m}^{3} / \text { month }\right)\end{array}$ & $\begin{array}{l}\text { Jumlah air recordedin } \\
\text { bill accounts } \\
\text { ( } \mathbf{m}^{3} / \text { month) }\end{array}$ & $\begin{array}{l}\text { GAP / difference } \\
\left(\mathrm{m}^{3} / \text { month) }\right.\end{array}$ \\
\hline 146.485 .24 & 109.863 .93 & 36.621 .31 \\
\hline
\end{tabular}

\section{F. Data water distribution}

Table 5. Water Distribusion

(Source : PDAM Surya Sembada Surabaya city )

With reference to the table of water usage patterns, then obtained the following calculation which will then be used to determine the level of water loss that occurred in PDAM Surya Sembada Surabaya on DMA 109: 
Table 6. Calculation Pattern usage of water

\begin{tabular}{|c|c|c|c|c|}
\hline Month & Stand Meter & Usage $\left(\mathrm{m}^{3} / \mathrm{month}\right)$ & $\begin{array}{l}\text { Average } \\
\left(\mathrm{m}^{3} / \mathrm{month}\right)\end{array}$ & Debit \\
\hline December 2015 & 4261.1 & & & \\
\hline January & 4286.7 & 25.6 & 24.875 & \\
\hline February & 4298.4 & 11.7 & 24.875 & \\
\hline Maret & 4321.6 & 23.2 & 24.875 & \\
\hline April & 4350.2 & 28.6 & 24.875 & \\
\hline May & 4382.4 & 32.2 & 24.875 & \\
\hline June & 4408.9 & 26.5 & 24.875 & \\
\hline July & 4421.3 & 12.4 & 24.875 & \\
\hline August & 4453.8 & 32.5 & 24.875 & \\
\hline September & 4480 & 26.2 & 24.875 & \\
\hline October & 4500.6 & 20.6 & 24.875 & \\
\hline November & 4532.1 & 31.5 & 24.875 & \\
\hline December & 4559.6 & 27.5 & 24.875 & \\
\hline Total & & 298.5 & & \\
\hline
\end{tabular}

(Source : Data research, 2017)

Calculation of water usage in 1 month with water meter reading ratio:

$=$ First Stand Meter - End Stand Meter

$=4286.7-4261.1$

$=25.6 \mathrm{~m}^{3} /$ month

With reference to the distribution table, we can calculate percent

water loss, calculated based on the

following Tornton formula :

$$
\begin{aligned}
& \quad H=\frac{D-K}{D} \times 100 \% \\
& \mathrm{D}=146.485 .24 \mathrm{~m}^{3} / \text { month } \times 12 \\
& =1.757 .822 .88 \mathrm{~m}^{3} / \text { year } \\
& \mathrm{K}=109.863 .93 \mathrm{~m}^{3} / \text { year } \times 12 \\
& =1.318 .367 .16 \mathrm{~m}^{3} / \text { year } \\
& H \\
& =\frac{1.757 .822 .88-1.318 .367 .16}{1.757 .822 .88} \times 100 \% \\
& \quad H=\frac{439.455 .72}{1.757 .822 .88} \times 100 \% \\
& =25 \% \\
& \text { Leakage in } \mathrm{m}^{3}=25 \% \times 1.757 .822 .88 \\
& \mathrm{~m}^{3}=439.455 .72 \mathrm{~m}^{3} / \text { year } \\
& =36.621 .31 \mathrm{~m}^{3} / \mathrm{month}^{2}
\end{aligned}
$$

calculation Q average :

$\mathrm{Q}=\frac{\text { amount of water }}{\text { amount of data }}=\frac{298,5}{12}=$ 24.875

$\frac{\text { Total difference of number water" meter" }}{\text { amount sample }} \times 100 \%$

$=1.220 .7 \mathrm{~m}^{3} /$ day

Calculates the percent value of water loss from the customer "meter"

$$
\frac{663.2}{3.326} \times 100 \%=19 \%
$$

Total water distribution $=146.485 .24$ $\mathrm{m}^{3}$

From lost water in "meter" distribusion customer and amount water distribution, we can calculation lost water:

146.485.24 $\mathrm{x} \quad 19 \%=27.832 .2$

$\mathrm{m}^{3} / \mathrm{month}=333.986 .4 \mathrm{~m}^{3} /$ year

Calculation of lost water in “ rupiah"

The total price of water sold is $=$ Rp. $184,131,946.68 \mathrm{~m}^{3} / \mathrm{month}=$ Rp 2,209,583,360.16 $\mathrm{m}^{3} /$ year 


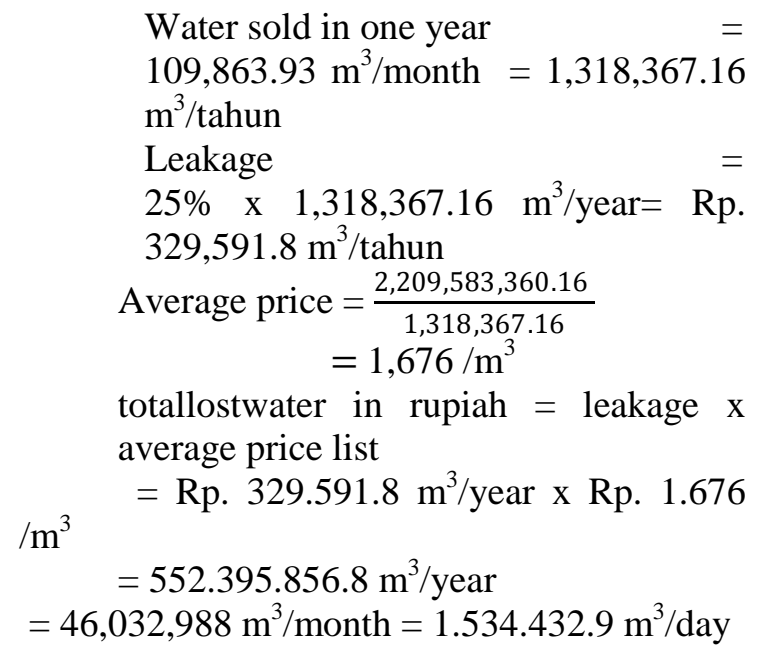

Water balance calculation:

- Water distribution at the month Januari - Desember $=146,485.24$ $\mathrm{m}^{3} /$ month $=1,757,822.88 \mathrm{~m}^{3} /$ year
- Consumption of metered yards

- $=109,863.93 \mathrm{~m}^{3} / \mathrm{month}=$ $1,318,367.16 \mathrm{~m}^{3} /$ year

- No accuracy customer "meter" = $27,832.2 \mathrm{~m}^{3} / \mathrm{month}=333,986.4$ $\mathrm{m}^{3} /$ year

- Lost water = amount water distribusion - consumsion acoount in meter tool $=1,757,822.88$ $1,318,367.16=439,455.72 \mathrm{~m}^{3} /$ year

- water loss

- = lost water - non technical loss= $439,455.72-333,986.4=$ $105,469.32 \mathrm{~m}^{3} /$ year

- Water balance calculation can be seen in the following table:

Water Balance Calculations

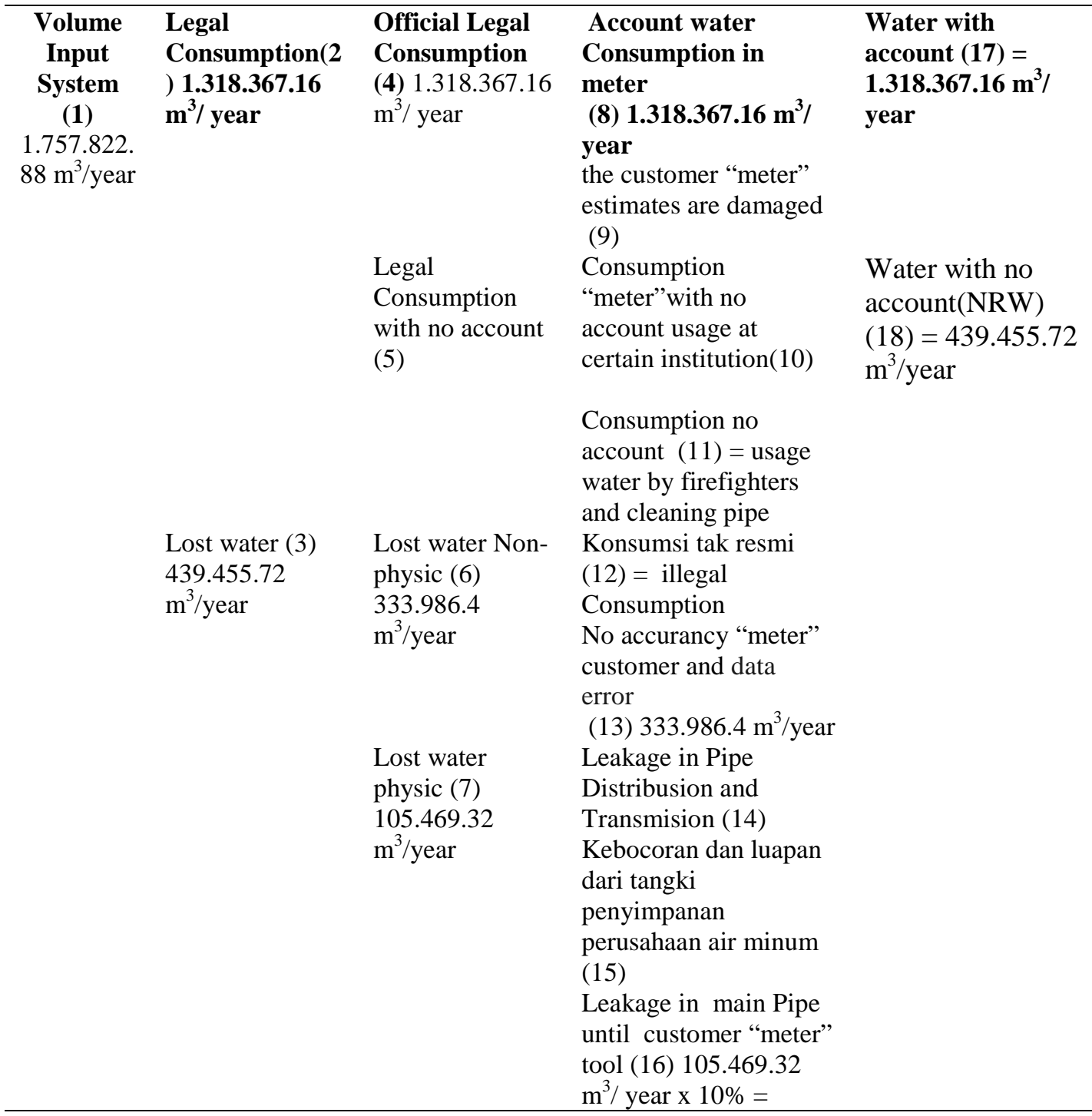


CalculateNon - Revenue Water (NRW) withInfrastructure Leakage Index (ILI) :Long main pipe $(\mathrm{LM})=5,580 \mathrm{~km}$.

Number of home connections

$(\mathrm{NC})=3.326$

Pressure average $(\mathrm{P})=2.04$

Average length of service pipes

$$
\begin{aligned}
& \text { (LP) }=15 \mathrm{~m} \\
& =0.015 \mathrm{~km}
\end{aligned}
$$

CAPL $(\mathrm{l} /$ year $)=$ physical loss at this time

$$
\begin{aligned}
& =374.592 .12 \mathrm{~m}^{3} / \text { year } \\
& =374.592 .120 \mathrm{l} / \text { year } \\
& \mathrm{LP}=0.015 \times 3.326 \\
& =49.89 \mathrm{~km}
\end{aligned}
$$

MAAPL $(\mathrm{l} /$ day $)=(18 \times$ LM $)+(0.8 \times$

$\mathrm{NC})+(25 \times \mathrm{LP}) \times \mathrm{P}$

$=(18 \times 5.580)+(0.8 \times 3.326)+(25 \times$ $49.89) \times 2.04$

$$
\begin{aligned}
& =(100,440+2,660.8+1.247 .25) \mathrm{x} \\
& 2.04 \\
& =104.308 .05 \times 2.04 \\
& =212.788 .422 \mathrm{l} / \mathrm{day} \\
& =6,383,652.66 \mathrm{l} / \mathrm{month} \\
& =76,603,831.92 \mathrm{l} / \mathrm{year} \\
& \text { ILI }=\frac{\text { CAPL }}{\text { MAAPL }} \\
& =\frac{374,592,120}{76,603,831.92} \\
& =4.88
\end{aligned}
$$

\section{Conclusion}

From the analysis of the discussion that has been described, it can be concluded as follows:

1. The cause of water loss in PDAM Surya Sembada Surabaya as follows:

a. Physical Water Loss:

- Reservoir leak

- Transmission pipe leaks

b. Non Physical Water Loss:

$10.546 .9 \mathrm{~m}^{3} /$ year
- Meter Error
- Wild Connection and Water Theft.

2. How to minimize water losses that occur in PDAM Surya Sembada Surabaya, can be controlled to control leaks - leaks that occur as follows :

- Establishment of zones and sub zones

- Establishment of sub zone to be monitored

- Assessment of distribution pipeline network conditions

- Checking the function and recording of water meters

- Sub zone stabilization

- Minimum Night Flow (AMM)

- Leak detection

\section{Acknowledgment}

This reseaches have been support from Dean UNIPA Surabaya and dan Chairman of the Industrial Engineering Department. In addition, thank you to the PDAM Surya Sembada Surabaya and Hendro Ardiyansyah have been helped and supported in this research.

\section{Reference}

1. Abbas, A.Z \& Ejah, U.S \& Gunadin, I.C \& Umar, M.H. (2014). Sistem Deteksi Kebocoran pada Jaringan Pipa Air PDAM Menggunakan Analisis Tekanan dengan Metode Extreme Learning Machine. Jurnal Tugas Akhir Teknik Informatika, D42110277.

2. Ardiansyah, H. 2014. Modul Sistem Distribusi, Surabaya.

3. Ardiansyah, Juwono, P.T \& Ismoyo, M.J. (2012). Analisa Kinerja Sistem Distribusi Air Bersih. Jurnal Teknik Pengairan, 3, 211 - 220.

4. Dharmasetawan, M. 2004. Sistem Perpipaan Distribusi Air Minum. Eka Mitra Engineering, Jakarta.

5. Farley, M. \& Wyeth, G. \& Istandar, A. \& Singh, S. (2008). The Manager's Non- Revenue Water Handbook : A 
TiBuana

Journal of applied Industrial Engineering-University of PGRI AdiBuana

Guide to Understanding Water Losses.

6. Hidayat, T. (2012). Pengurangan Tingkat Kehiangan Air Melalui Perbaikan Meteran Air dan Penggantian Meteran Air yang Hilang dengan Pendekatan Sistem Dinamik. Jurnal Teknik Industri, 4 - 5, 978 - 979.

7. Kementrian Pekerjaan Umum. 2009. Pedoman Penurunan Air Tak Berekening (ATR), Jakarta.

8. Malabay. (2008). Pendekatan Sistem Model Causal Loop Diagram (CLD) Dalam Memahami Permasalahn Penerimaan Kuantitas Mahasiswa Baru Di Perguruan Tinggi Swasta. Pendekatan Model Causal Loop Journal, 1411 - 6286.

9. Rita, D.K. \& Nugraha, W.D. (2010). Studi Kehilangan Air Akibat Kebocoran Pipa Pada Jalur Distribusi PDAM Kota Magelang (Studi Kasus : Perumahan Armada Estate dan Depkes, Kramat Utara Kecamatan Magelang Utara). Jurnal
PRESPITASI, 7(2) : Program Studi Teknik Lingkungan FT UNDIP.

10. Sarungallo, G.A \& Wardhani,E. (2016). Evaluasi Sistem Jaringan Distribusi Air Bersih di Kecamatan Pontianak Selatan Kota Pontianak Provinsi Kalimantan Barat. Jurnal Rekayasa Lingkungan, 4, 1.

11. Siregar, N.A \& Mulia, A.P .(2012). Evaluasi Kehilangan Air (Water Losses) PDAM Tirtanadi Padangsidimpuan di Kecamatan Padangsidimpan Selatan.

12. Tahid, Suwarno \& Nurcahyanie, Yunia Dwie. (2007). Konsep Teknologi dalam Pengembangan Produk Industri Pendekatan Kolaboratif pada Konsep Teknologi dan Desain Produk Industri. Jakarta: Kencana.

13. Tornton dkk. 2008. Penurunan Kehilangan Air, Semarang.

14. Yayasan Pendidikan Tirta Dharma. 2006. Modul D.32 Kehilangan Air Fisik, Magelang. 